\title{
Comparison of Pinhole Collimation and Focusing Optics for SANS
}

\author{
C.J. Glinka ${ }^{1,2, *}$, J.G. Barker ${ }^{2}$ and D.F.R. Mildner ${ }^{2}$ \\ ${ }^{1}$ University of Delaware, Newark, DE 19716 \\ ${ }^{2}$ National Institute of Standards and Technology, Gaithersburg, MD 20899 \\ * Corresponding author. Tel: +1 301975 6242, Email: cglinka@nist.gov
}

\begin{abstract}
We examine quantitatively the instrumental factors that affect the theoretical performance and practical application of conventional pinhole collimation and focusing optics for small-angle neutron scattering (SANS) measurements. We calculate the relative performance of pinhole collimation vis-à-vis focusing by grazing incidence elliptical mirrors and compare the results with a recent ray-tracing simulation to show that the performance gains due to focusing found in the simulation arise largely from assuming a much larger sample size. We also compare measurements of the parasitic scattering from pinhole collimation with that from focusing cylindrical quartz mirrors, and a focusing refractive optic, to stress the importance of signal-tonoise as the true measure of performance for SANS instruments.
\end{abstract}

Keywords: neutron scattering; focusing optics; small-angle scattering; grazing incidence mirrors; parasitic scattering; pinhole collimation

\section{Introduction}

The development of cold neutron sources, neutron guides and large area neutron detectors in the early 1970's has led to a tremendous expansion in the application of small-angle neutron scattering (SANS) to the study of materials microstructure worldwide. Another essential development has been the addition of neutron guide halls to existing reactor buildings enabling the construction of SANS instruments with long flight paths and large beams to satisfy the conflicting requirements for low beam divergence with high scattered beam intensity. These instruments operate essentially like pinhole cameras with "pinhole" apertures up to several cm in diameter spaced up to tens of meters apart in evacuated flight paths to provide the necessary beam collimation with high beam current on the sample. Equally good collimation of 
the scattered beam is achieved by positioning a large two-dimensional detector at a comparable distance from the sample.

There have been a number of attempts to improve the performance of SANS instruments through the use of devices for focusing neutrons. These have included multi-beam converging pinhole collimation [1-3], multiple refractive lenses [4, 5], magnetic lenses [6, 7] and grazing incidence mirrors $[8,9]$. The gains reported or projected for such devices, in terms of increased beam current or scattered intensity for fixed angular resolution, have varied from a few (2-5) to orders of magnitude. This wide range in reputed performance begs the question as to what are the fundamental factors that determine the advantages and limitations of various types of focusing techniques. Here we address this question by examining in detail the potential advantages, and limitations, of focusing optics vis-à-vis traditional simple pinhole collimation for SANS.

We begin by comparing pinhole collimation with an idealized, non-specific focusing device to get a sense of where the potential benefits and limitations originate. We wish to compare the beam current, I (neutrons per second), on the sample for instrument configurations with the same angular resolution, where

$$
I=\frac{d^{2} \phi_{s}}{d \Omega d \lambda} \Delta \lambda \frac{A_{1} A_{2}}{L_{1}^{2}}
$$

and $d^{2} \phi_{s} / d \Omega d \lambda$ is the source brightness, $\mathrm{A}_{1}$ is the area of the neutron source viewed by the instrument, $L_{1}$ is the source-to-sample distance, $A_{2}$ is the area of the sample, and $\Delta \lambda$ is the wavelength spread in the incident beam. Thus $A_{1} / L_{1}^{2}=\Delta \Omega_{s}$ is the solid angle of the source with respect to the sample, so that $I / A_{2}$ is the neutron flux $\left(\mathrm{n} / \mathrm{cm}^{2} / \mathrm{s}\right)$ at the sample.

Figure 1 shows the layout of a conventional pinhole collimation SANS instrument. For a given angular resolution (defined below) and when the total instrument length is constrained $L_{1}+L_{2}=$ constant, the beam current is optimized when $L_{1}=L_{2}$, and the source pinhole radius is twice that of the sample, $R_{1}=2 R_{2}[16]$. In this case the beam profile on the detector is a truncated cone [17] (approximately triangular in cross section) with a base radius $R_{d}=2 R_{1 p}$. The 
55 minimum accessible scattering angle for this configuration is $\Theta_{\min }=R_{d} / L_{2}$, corresponding to

56 a minimum scattering vector $Q_{\min }$,

57

$$
Q_{\min }=k \Theta_{\min }=k \frac{R_{d}}{L_{2}}=k \frac{2 R_{1 p}}{L_{2}}
$$

58

59

60

61

62 Figure 2 shows the layout of an ideal focusing optic SANS instrument. Here the optic is

63 idealized as a perfectly focusing thin lens that images the source aperture onto the neutron

64

65

66

67

68

69

70

71

72

73

74 For a given $Q_{\min }$, the gain provided by an ideal focusing optic compared with pinhole collimation 75

76

where $k=2 \pi / \lambda$. Using equation [2], the beam current [1] can be rewritten as

$$
I_{p}=\frac{d^{2} \phi_{s}}{d \Omega d \lambda} \Delta \lambda\left(\frac{\pi}{4}\right) A_{2 p}\left(\frac{Q_{\min }}{k}\right)^{2}
$$

where the subscript $\mathrm{p}$ refers to the pinhole instrument.

$\ll<<$ Figure $1 \gg \gg>$ detector with unit magnification. In this case, the beam size on the detector is independent of the sample size and is determined only by the source aperture radius, $R_{1}$. Thus $Q_{\min }$ and the beam current are given by

$$
Q_{\min }=k \frac{R_{1 L}}{L_{2}}
$$

and

$$
I_{L}=\frac{d^{2} \phi_{s}}{d \Omega d \lambda} \Delta \lambda \pi A_{2 L}\left(\frac{Q_{\min }}{k}\right)^{2}
$$

where $A_{2 L}$ is the area of the sample illuminated by the optic. Note that [5] is correct for any magnification $M=L_{2} / L_{1}$, since the image of the source has a radius $\left(L_{2} / L_{1}\right) R_{1}$, so that $Q_{\min }=$ $k R_{1} / L_{1}$ for all M. This point is discussed more fully in [18].

$\ll<<$ Figure $2>\gg>$ can be defined as the ratio of the beam currents on the sample. From equations [2-5], the gain is 


$$
\operatorname{Gain}\left(\text { fixed } Q_{\text {min }}\right)=\frac{I_{L}}{I_{p}}=\frac{A_{1 L}}{A_{1 p}} \frac{A_{2 L}}{A_{2 p}}=4 \frac{A_{2 L}}{\pi R_{2 p}^{2}}
$$

78

79

80

81

since $R_{1 L}=2 R_{1 p}$ for the same $Q_{\text {min. }}$. The factor of 4 comes from the gain in flux (current/sample area, also called the fluence rate) at the sample.

Another approach would be to compare beam currents for a fixed variance in the scattering vector, $\sigma_{Q}^{2}$, which gives

$$
\text { Gain (fixed variance) }=2 \frac{A_{2 L}}{\pi R_{2 p}^{2}}
$$

where variances are calculated as in [16, 18]. Equations [6] and [7] show that an ideal focusing optic can increase the flux at the sample by at most a factor of 4 , for fixed $Q_{\min }$, or by 2 , for fixed variance in $\mathrm{Q}$. Much larger gains can be achieved in the beam current at the sample with a focusing optic because significantly larger sample sizes may be used without affecting the Qresolution. For pinhole collimation, the optimal sample area is $1 / 4^{\text {th }}$ the area of the source aperture (when $L_{1}=L_{2}$ ), whereas with focusing the sample area can be considerably larger, potentially providing much larger gains in beam current.

\section{Focusing by Grazing Incidence Mirrors}

Neutrons will undergo total reflection from a smooth surface for angles of incidence that are less than a critical angle $\Theta_{c}=\gamma_{c} \lambda$, where $\gamma_{c}$ depends only on the material of the reflecting surface $^{1}$. For $\mathrm{Ni}$, one of the best reflecting elements, $\gamma_{\mathrm{c}}=0.017 \mathrm{rad} / \mathrm{nm}$, or $\approx 1.0$ degrees $/ \mathrm{nm}$. For $5 \AA$ A neutrons, $\Theta_{c} \approx 0.5$ degrees for a Ni surface. Thus as for X-rays, grazing incidence mirrors can be used to focus neutrons.

Consider, for example, an ellipsoidal mirror equidistant from its two focal points as shown in Fig. 3. In this position the mirror is analogous to the ideal optic depicted in Fig. 2 in that it images a neutron source at one focal point of the ellipsoid onto a detector at the other focus

\footnotetext{
${ }^{1} \gamma_{c}=\sqrt{n b / \pi}$ where $\mathrm{n}$ is the number of atoms per unit volume and $\mathrm{b}$ is the coherent scattering amplitude of the reflecting medium.
} 
101

$$
I_{m} \cong \frac{d \phi_{s}}{d \Omega d \lambda} \Delta \lambda \frac{2 L_{m}}{\sqrt{a^{2}-b^{2}}}\left(\frac{\pi b}{1+X_{m}}\right)^{2}\left(\frac{1-X_{m} / 2}{1+X_{m} / 2}\right)^{2}\left(\frac{Q_{\min }}{k}\right)^{2}
$$

117 From [2], [3] and [12], the ratio of the beam current on the sample for an ideal ellipsoidal

with a magnification of unity. For the geometry shown in Fig. 3, the solid angle of the source aperture with respect to the sample is approximately,

$$
\Delta \Omega_{S} \cong \frac{\pi R_{1 m}^{2}}{\left(L_{1}+L_{m}\right)^{2}}
$$

For SANS the optimal location for the sample would be as close to the mirror as possible to maximize $L_{2}$. At this location the optimal sample geometry is a thin ring with an area, $A_{s}$, given approximately by

$$
A_{S} \cong \frac{2 \pi b^{2} L_{m}}{\sqrt{a^{2}-b^{2}}}
$$

where $a$ and $b$ are the semi-major and semi-minor axes of the ellipse in Fig. 3. $Q_{\min }$ for the configuration in Fig. 3 is

$$
Q_{\min }=k \frac{B_{S}}{L_{2}}
$$

where $B_{s}$ is the radius of the beam spot on the detector. Ideally, $B_{s}=\mathrm{R}_{1 \mathrm{~m}}$, however, for a long mirror, as needed to illuminate a large sample area, the effect of coma should be taken into account leading to a better estimate for $Q_{\min }$ given by

$$
Q_{\min }=k \frac{R_{1 m}}{L_{2}}\left(\frac{1+X_{m} / 2}{1-X_{m} / 2}\right)
$$

where $X_{m}=L_{m} / L_{2}$.

With the expressions in equations [8], [9] and [11], the beam current on the sample [1] can be expressed as

$$
\text { mirror vis-à-vis single pinhole collimation for the same } Q_{\min } \text { and the same overall instrument }
$$


length, ( $L_{T}=L_{1}+L_{2}$ for pinhole collimation and $L_{T}=L_{1 m}+L_{m}+L_{2 m}$ for the mirror instrument) and wavelength band becomes

$$
\frac{I_{m}}{I_{p}} \cong\left(\frac{4}{\pi A_{2 p}}\right) \frac{2 L_{m}}{\sqrt{a^{2}-b^{2}}}\left(\frac{\pi b}{1+X_{m}}\right)^{2}\left(\frac{1-X_{m} / 2}{1+X_{m} / 2}\right)^{2}
$$

Note that in the limit where $L_{m} \rightarrow 0$, Eq. 13 is equivalent to Eq. 6 if $A_{2 L}$ is set equal to area of annulus that accepts reflection from the mirror. To understand how large this ratio of beam currents can be, in principle, consider two SANS instruments each with a total length, $L_{T}=30 \mathrm{~m}$, operating at the same wavelength of $6.28 \AA\left(k=1 \AA^{-1}\right)$ and configured for a $Q_{\min }$ of $0.001 \AA^{-1}$. For a pinhole collimation instrument, this implies source and sample apertures with radii of $0.75 \mathrm{~cm}$ and $0.375 \mathrm{~cm}$, respectively, from equation [2]. We compare this with a SANS instrument with a $50 \mathrm{~cm}$ long $\left(L_{m}\right)$ ellipsoidal Nickel mirror with a semi-major axis, $a=15 \mathrm{~m}$, and a semi-minor axis, $b=15.7 \mathrm{~cm}$. For this choice of axes, the angles of incidence for the neutrons impinging on the mirror are less than the critical angle at this wavelength and thus satisfy the condition for total reflection. For this length of mirror, $X_{m}=\mathrm{L}_{m} / \mathrm{L}_{2 m}=0.034$ and the effects of coma are quite small. For these sets of values for the two instruments, the ratio of beam currents $I_{m} / I_{p}=400$ from [14]. This gain, while impressive, is due primarily to the much larger sample area implied by the mirror geometry in Fig. 3. The sample area for the pinhole instrument, $\mathrm{A}_{\mathrm{p}}=\pi R_{2 p}^{2}$, would be $0.44 \mathrm{~cm}^{2}$, whereas the sample for the mirror instrument is an annular ring with an area, $A_{m} \approx$ $52 \mathrm{~cm}^{2}$. Hence, $A_{m} / A_{p} \approx 120$ and accounts for all but a factor of 3.3 (due to the gain in flux) of the gain in beam current on the sample. Thus large gains in beam current require large samples with an annular geometry that may not be practical in many cases. Nevertheless, significant gains are possible depending on how much of the available sample area can be utilized for a particular measurement.

$<<<$ Figure $3>>>>$

\section{Comparison with Pinhole Collimation}

A comparison of the simulated performance of a SANS instrument using a grazing incidence ellipsoidal mirror with that of a simple pinhole instrument has been carried out by Liu, et al. [10]. The simulation was performed by ray tracing for instruments with the same overall length 
147 (13 $\mathrm{m})$ operating with the same wavelength (13 $\AA$ ). A complete list of the parameters used in the simulation is given in Table 1.

Table 1. Rows 1 and 2 are the parameters for the pinhole collimation and focusing nickel ellipsoidal mirror SANS instruments simulated by Liu et al. $L_{1}$, and $L_{2}$ are the source-to-sample and the sample-to-detector distances, respectively. $R_{1}$ is the radius of the source aperture, $A_{2}$ is the area of the beam at the sample position (maximum sample area), and $R_{d}$ is the nominal radius of the beam at the detector (i.e. calculated, not simulated). For the mirror instrument, the beam area at the sample is an annulus with a mean radius of $8.0 \mathrm{~cm}$ and a width of $6 \mathrm{~mm}$. The third row has parameters for an optimized pinhole SANS instrument with the same $Q_{\min }$ and overall length of $13 \mathrm{~m}$. The last two columns are the flux $\left(\mathrm{n} / \mathrm{cm}^{2}-\mathrm{s}\right)$ and the beam current $(\mathrm{n} / \mathrm{s})$ at the sample relative to that for the short pinhole collimation SANS instrument. The wavelength for calculating $Q_{\min }$ is $13 \AA$ in every case.

\begin{tabular}{|c|c|c|c|c|c|c|c|c|c|c|}
\hline Instrument & $\begin{array}{l}\mathrm{L}_{1} \\
(\mathrm{~m})\end{array}$ & $\begin{array}{l}\mathrm{L}_{2} \\
(\mathrm{~m})\end{array}$ & $\begin{array}{l}R_{1} \\
(\mathrm{~mm})\end{array}$ & $\begin{array}{l}R_{2} \\
(\mathrm{~mm})\end{array}$ & $\begin{array}{l}A_{2} \\
\left(\mathrm{~cm}^{2}\right)\end{array}$ & $\begin{array}{l}\mathbf{R}_{\mathbf{d}} \\
(\mathrm{mm})\end{array}$ & $\mathbf{R}_{\mathrm{d}} / \mathrm{L}_{\mathbf{2}}$ & $\begin{array}{l}\mathbf{Q}_{\min } \\
\left(\AA^{-1}\right)\end{array}$ & $\begin{array}{l}\text { Flux } \\
\left(\mathrm{cm}^{2} \mathrm{~s}^{-1}\right)\end{array}$ & $\begin{array}{l}\mathrm{I}\left(\mathrm{s}^{-1}\right) \\
\text { sample }\end{array}$ \\
\hline $\begin{array}{l}\text { Short } \\
\text { pinhole }\end{array}$ & 4 & 9 & 5 & 5 & 0.79 & 27.5 & 0.0031 & 0.0015 & 1 & 1 \\
\hline $\begin{array}{l}\text { Focusing } \mathrm{Ni} \\
\text { mirror ( } 0.4 \\
\text { m long) }\end{array}$ & 4 & 9 & 5 & $\mathrm{~N} / \mathrm{A}$ & 30. & 11.3 & 0.0013 & 0.0006 & 1.3 & $\approx 50$ \\
\hline $\begin{array}{l}\text { Optimized } \\
\text { short } \\
\text { pinhole }\end{array}$ & 6.5 & 6.5 & 10 & 5 & 0.79 & 20 & 0.0031 & 0.0015 & 1.5 & 1.5 \\
\hline
\end{tabular}

For the chosen parameters for the two instruments, Liu et al. find from their simulation that the beam current ( $\mathrm{n} / \mathrm{sec}$ for the entire available sample area) is $\approx 50$ times greater for the focusing instrument compared to the pinhole instrument. This large gain is, as Liu et al. point out, largely due to the much larger beam area at the sample (38 times larger, as seen in the table) for the focusing instrument. Hence much larger sample volumes (38x) would be necessary to realize a comparable gain in scattered intensity.

We also point out that Liu et al.'s comparison is with a non-optimized pinhole SANS instrument. An optimized instrument of the same overall length would have $L_{1}=L_{2}$, and $R_{1}=2 R_{2}$. The third 
row of Table 1 gives the parameters for an optimized instrument having the same $Q_{\min }$ as the pinhole instrument in Liu et al.'s simulation. The flux at the sample for the optimized pinhole instrument is $50 \%$ higher, and is, in fact, greater than that for the focusing mirror instrument. The total available beam current is still significantly greater for the focusing instrument $(\approx 30)$, but requires $38 x$ more sample.

To be fair, a comparison of a focusing mirror SANS with a pinhole SANS should be made for instruments configured for the same $Q_{\min }$. This is not the case for the configurations in Table 1 where the nominal $Q_{\min }$ for an ideal (no aberrations) mirror SANS is less than half that for the pinhole SANS. However, in their actual ray-tracing simulation for the focusing mirror SANS, Liu et al. show a minimum-Q (Fig. 1 in ref. 1) of about $0.0015 \AA^{-1}$, not very different from their simulation of a pinhole SANS which gave a $Q_{\min }$ of about $0.002 \AA^{-1}$.

\section{Signal-to-Noise Considerations}

While increasing the beam current at the sample is important, equally important to the performance of a SANS instrument is the signal-to-noise ratio achievable at the low-Q limit for a particular instrument. Here noise refers to whatever signal (neutrons, gamma rays or electronic noise) occurs at a particular Q-value that is not due to scattering from the sample. The most pernicious source of noise for a SANS instrument is the so-called parasitic scattering, from the edges of slits or apertures in the case of pinhole instruments or from non-specular scattering in the case of grazing incidence mirrors, since both types of parasitic scattering tend to peak in the forward direction $(Q=0)$. An underappreciated feature of pinhole collimation SANS instruments is the relative ease of fabricating apertures (with beveled edges to eliminate edge reflections) in highly absorbing, machineable materials such as cadmium and gadolinium that minimize parasitic scattering. The apertures in a long-flight-path SANS instrument are correspondingly large, resulting in a low ratio of edge area to aperture area, which further mitigates the adverse effects of parasitic scattering. Grazing incidence mirrors, on the other hand, have an inherently large surface area which can contribute to the non-specular scattering from surface roughness that shows up as a halo around the main beam at the detector. 
Sources of parasitic scattering are difficult to simulate, and are not included at all in the simulations done by Liu et al. Measurements must be carried out to assess its importance in any proposed SANS instrument. Figure 4 shows an example of such a measurement for a pinhole SANS instrument. These data were taken on one of the 30-m SANS instruments at the NCNR (on guide NG-B in the expanded guided hall). The neutron wavelength was $8.4 \AA$ with a wavelength spread, $\Delta \lambda / \lambda=13 \%$. To minimize sources of parasitic scattering the data were taken with the entire 30-m length of the instrument under vacuum ( $P \approx 0.1$ Torr); hence there were no beam windows between the source pinhole aperture $\left(R_{1}=12.7 \mathrm{~mm}\right)$ and the instrument's $64 \mathrm{~cm}^{2} \times 64 \mathrm{~cm}^{2}$ position-sensitive detector, a He-3 proportional counter with $5 \mathrm{~mm} \times 5 \mathrm{~mm}$ spatial resolution. The sample aperture was a circular hole in $0.5 \mathrm{~mm}$ thick cadmium sheet with a radius $R_{2}=6.4 \mathrm{~mm}$. The source-to-sample aperture distance was $\mathrm{L}_{1}=$ $1627 \mathrm{~cm}$ and the detector was located at its farthest distance from the sample, $\mathrm{L}_{2}=1317 \mathrm{~cm}$. For this configuration, the calculated minimum-Q value is $0.0018 \AA^{-1}$. The beam profile measurement was carried out in two steps. First, calibrated attenuators were inserted in the beam upstream from the source aperture to allow the beam stop in front of the detector to be removed in order to measure the intense inner part of the profile. Then, with the beam stop (50 $\mathrm{mm}$ in diameter) in place and the attenuators removed, the wings of the profile were measured. A background measurement was also made, with the beam blocked at the sample position, and subtracted from both of these measurements to correct for all sources of background not associated with the beam itself (e.g. electronic noise, room background, etc.). Also plotted in Fig. 4 is a calculation of the contribution to the profile due to Fraunhofer diffraction from the edges of the sample aperture (see Appendix A for details).

The data in Fig. 4 show that there is some parasitic scattering over and above that expected from Fraunhofer diffraction. The source of this extra parasitic scattering is at present unknown. Nevertheless, even with this extra scattering, the signal-to-noise achieved with this simple setup is over $10^{5}$ and more than sufficient to measure weak scattering signals from actual samples from the calculated $Q_{\min }$ to the edges of the detector. 
Measurements directly comparable to pinhole collimation beam profile measurements, similar to those in Fig. 4, for grazing incident metallic mirrors of the type proposed by Liu and others (i.e. fabricated by an electroformed replication process (ERP)) have to our knowledge not been published. The development of these mirrors has focused thus far on neutron imaging applications where non-specular scattering is less of a concern.

To gain some insight into the effects of non-specular scattering on signal-to-noise for SANS, we have measured the specular and non-specular scattering from fused silica optical glass (zerodur) substrates with highly polished cylindrical surfaces fabricated by F. Cooke Inc., North Brookfield, $\mathrm{MA}^{2}$. The root-mean-square surface roughness of the mirrors, measured with an optical profilometer [11], ranged from $3 \AA$ to $10 \AA$. . The mirrors were made from substrates 30 $\mathrm{cm}$ long, by $5 \mathrm{~cm}$ wide, by $2.5 \mathrm{~cm}$ thick that were ground and polished with a radius of curvature (perpendicular to the $5 \mathrm{~cm}$ width), corresponding to the semi-minor axis dimension in Fig. 3, of $13 \mathrm{~cm}$. Each mirror was carefully aligned and rotated about a horizontal axis perpendicular to the beam by $0.45^{\circ}$ to reflect vertically (like a plane mirror) and focus horizontally (with unity magnification) a beam of $9 \AA$ neutrons onto the instrument's detector $\left(L_{2}=15 \mathrm{~m}\right)$. This arrangement corresponds to having the mirror at the lower position of the mirrors shown schematically in Fig. 3, with $\mathrm{L}_{m}=30 \mathrm{~cm}$. A cadmium mask with a slit aperture $(25$ $\mathrm{mm}$ wide $\mathrm{x} \approx 2.5 \mathrm{~mm}$ high) was affixed to the front of the mirror to allow full illumination (at the angle of incidence of $0.45^{\circ}$ ) of only the reflecting surface. The measurements were made at the NG-7, $30 \mathrm{~m}$ SANS instrument at NIST's NCNR. For this configuration, the $25 \mathrm{~mm} \times 2.5 \mathrm{~mm}$ beam at the mirror formed a specular peak at the detector that was $\approx 14 \mathrm{~mm}$ wide (due to the focusing in the horizontal plane) and $\approx 18 \mathrm{~mm}$ high (due to plane mirror reflection in the vertical direction). For comparison, measurements were also made with no mirror but with a 3 $\mathrm{mm}$ circular pinhole at the sample which gave a circular beam profile at the detector $\approx 19 \mathrm{~mm}$ in diameter. The calculated minimum $\mathrm{Q}$ for both the mirror and pinhole configurations was $\approx$

\footnotetext{
${ }^{2}$ Certain commercial equipment, instruments, or materials are identified in the text in order to adequately describe the experimental procedures. Such identification does not imply recommendation or endorsement by NIST.
} 
$0.00068 \AA^{-1}$. Figure 5 shows the results of these measurements, again along with the calculated Fraunhofer diffraction from a $3 \mathrm{~mm}$ diameter circular aperture.

$$
\ll<<\text { Figure } 5>>>>
$$

The data in Fig. 5 point out the difficulty of achieving good signal-to-noise in SANS as measurements are pushed to lower and lower $\mathrm{Q}$-values. The signal-to-noise at $\mathrm{Q}_{\min }$ for the best mirror tested, with rms surface roughness of $\approx 3 \AA$, is similar to that achieved with the small pinhole, but is only about $10^{4}$. The signal-to-noise for the poorer mirror is only about $10^{3}$. The reflected beam current $(\mathrm{n} / \mathrm{s})$ for the mirrors is about 15 times greater than for the pinhole, due largely to the larger beam area at the sample position ${ }^{3}$. The parasitic scattering from the pinhole is even larger, compared with the calculated Fraunhofer diffraction, than that observed in the data in Fig. 4. This may be due in part to having windows and an air path in the sample region that would have contributed to the background for both the mirrors and pinhole.

We note the similarity in the Q-dependence of the parasitic scattering for the pinhole and mirrors in Fig. 5, which is unexpected. It appears that the actual non-specular scattering from the mirrors is relatively Q-independent in this limited Q-range and adds to the Q-dependent scattering seen in the pinhole data. The $Q$-dependence of the pinhole scattering is similar to that expected from Fraunhofer diffraction $\left(\propto Q^{-3}\right)$, but is more than an order of magnitude greater.

A focusing optic can outperform pinhole collimation when small apertures are required to reach a very low $Q_{\min }\left(<0.001 \AA^{-1}\right.$ for the 30 m SANS instruments at the NCNR) as has been pointed out by other authors $[9,14]$. For this reason the NCNR's instruments do use a focusing optic to achieve their best low-Q performance [5]. This device focuses by refraction and consists of multiple biconcave spherical lenses of $\mathrm{MgF}_{2}$ which focus an $8.4 \AA$ beam, $\approx 13 \mathrm{~mm}$ in diameter at the sample, onto the detector $\left(L_{2} \approx 15 \mathrm{~m}\right)$. Figure 6 shows the beam profile for this lens system together with the data from Fig. 5 for the better cylindrical mirror for comparison.

$\ll<<$ Figure $6>\gg>>$

\section{Discussion and Conclusions}

In the preceding sections, we have shown that significant gains in performance of focusing mirrors vis-à-vis pinhole collimation for SANS depend almost entirely on the use of relatively large samples, since sample size does not, in principle, impact Q-resolution. Potential gains in flux at the sample range from 2 to 4 , depending on the criterion used for Q-resolution, and any

\footnotetext{
${ }^{3}$ The increase in beam current at the sample is not evident in Fig. 5 since the data have been normalized by dividing by the beam current (see Appendix B for details).
} 
additional gain in signal (neutrons scattered per second) comes from a larger sample size, which may also be required to have a non-standard shape (e.g. annular rather than circular or square).

Based on these results and past experience, we foresee the main utility of using focusing optics for SANS is in extending the measurement range of pinhole collimation to lower Q-values where the sample size must be decreased using pinhole collimation, not in supplanting pinhole collimation. To be effective this option requires an optic with minimal non-specular scattering and a high resolution detector to match the focusing characteristics of the optics. An example of such a mirror focusing SANS instrument is the KWS-3 $[9,19]$.

The parasitic scattering we did observe in our measurements on pinholes, cylindrical focusing mirrors and the refractive lens system currently in use on the $30 \mathrm{~m}$ SANS instruments at the NCNR is presently not well understood. The pinhole scattering is significantly greater than our estimate of Fraunhofer diffraction from aperture edges. Interestingly, the Q-dependence of the scattering from the pinholes, and from the mirrors and lenses, is similar to that from Fraunhofer diffraction, having an approximately $\mathrm{Q}^{-3}$ behavior.

\section{References}

1. A.C. Nunes, (1978). J. Appl. Cryst. 2, 460-464.

2. C.J. Glinka, J.M. Rowe, J.G. LaRock, J. Appl. Cryst. 19 (1986) 427-439; P. Thiyagarajan, et al., J. Appl. Cryst., 30, (1997) 280-293.

3. A. Brulet, V. The' venot, D. Lairez, S. Lecommandoux, W. Agut, S.P. Armes, Du J. Jianzong, S. Dé sert, S. (2008). J. Appl. Cryst., 41, 161-166.

4. M.R. Eskildsen, P.L. Gammel, E.D. Isaacs, C. Detlefs, K. Mortensen, D. Bishop, Nature 391 (19) 563-566.

5. S.-M. Choi, J.G. Barker, C.J. Glinka, Y.T. Cheng, P.L. Gammel, J. Appl. Cryst.. 33 (1), (2000) 793796.

6. M. Yamada, Y. Iwashita, M. Ichikawa, T. Sugimoto, H. Tongu, H. Fujisawa, H.M. Shimizu, T. Ino, K. Mishima, K. Taketani, Physica B 404 (2009) 2646-

7. M. Yamada, Y. Iwashita, T. Kanaya, J.L. Yamada, H.M. Shimizu, Physica B 406 (2011) 2453-

8. B. Alefeld, D. Schwahn, T. Springer, Nuclear Instruments and Methods in Physics Research A 274 (1989) 210

9. E. Kentzinger, L. Dohmen, B. Alefeld, U. Rücker, J. Stellbrink, A. loffe, D. Richter, Th. Brückel, Physica B 350 (2004) e779-e781.

10. D. Liu, M.V. Gubarev, G. Resta, B.D. Ramsey, D.E. Moncton, B. Khaykovich, Nuclear Instruments and Methods in Physics Research A 686 (2012) 145-150.

11. P. Z. Takacs, S. C. K. Feng, E. L. Church, S. N. Qian, W. M. Liu, Vol. 966, SPIE, 1988. 
12. C. J. Glinka, J. G. Barker, B. Hammouda, S. Krueger, J. J. Moyer, W. J. Orts, Journal of Applied Crystallography 31, 430-445 (1998).

13. J.G. Barker, C.J. Glinka, (1992) Proceedings of the Society of Photo-Optical Instrumentation Engineers (SPIE) 1738, 386-394, Edited by: C.F. Majkrzak and J.L. Wood.

14. B. Alefeld, L. Dohmen, D. Richter, Th. Brückel, Physica B 283 (2000) 330-332.

15. G. B. Airy, Trans. Cambridge Phil. Soc. 5 (1835) 283.

16. D.F.R. Mildner, J.M. Carpenter, Journal of Applied Crystallography 17, 249-256 (1984).

17. J. G. Barker, J. S. Pedersen, Journal of Applied Crystallography 28, 1-05-114 (1995).

18. D.F.R. Mildner, J. Appl. Cryst. 47, 1247-1251 (2014).

19. G. Goerigk, Z. Varga, J. Appl. Cryst. 44, 337-342 (2011).

\section{Acknowledgements}

This work utilized facilities supported in part by the National Science Foundation under Agreement No. DMR-0944772

\section{Appendix A}

The diffraction pattern produced by a plane wave incident on a circular aperture is given by the Airy function [15]. In the limit that the scattering angle $\theta$ is small such that $\sin (\theta) \approx \theta$ the Airy solution simplifies to

$$
\frac{d S}{d \Omega}(Q)=\frac{\pi R_{2}^{2}}{\lambda^{2}}\left[\frac{2 J_{1}\left(Q R_{2}\right)}{Q R_{2}}\right]^{2}
$$

where $J_{1}$ is the first order Bessel function, $R_{2}$ is the radius of the aperture, and $d S / d \Omega$ is the probability that a neutron is scattered per steradian. When $Q R_{2} \gg 1$, which is the case for the pinhole data in Figs. 4, 5 and 6, equation A1 can be approximated as

$$
\frac{d S}{d \Omega}(Q) \approx \frac{4}{R_{2} \lambda^{2}} Q^{-3}
$$

It is equation A2 that is plotted as the dashed lines in Figs. 4, 5 and 6.

\section{Appendix B}

In figures 4, 5 and 6 we have chosen to plot beam profile and attendant parasitic scattering data in terms of the probability that a neutron is scattered per steradian, $d S / d \Omega$, where

$$
\frac{d S}{d \Omega} d \Omega=\frac{\# \text { neutrons per sec scattering into } d \Omega}{\# \text { neutrons per sec incident on sample }}=\frac{I(Q) d \Omega}{I_{S}}
$$


where $I_{s}$ is the beam current at the sample (equation [1]). Defined this way, and including the entire beam profile in $\mathrm{I}(\mathrm{Q}) \mathrm{d} \Omega, \int_{0}^{4 \pi} \frac{d s}{d \Omega} d \Omega=1$. In measurements on actual samples, $\mathrm{I}(\mathrm{Q})$ is related to the cross section per unit volume (i.e. the macroscopic cross section) by

$$
I(Q) d \Omega=I_{s} T d \frac{d \Sigma}{d \Omega} d \Omega
$$

where $T$ is the transmission of the sample, $d$ is the thickness of the sample, and $d \Sigma / d \Omega$ is the macroscopic cross section for the sample. Hence

$$
\frac{d S}{d \Omega}=T d \frac{d \Sigma}{d \Omega}
$$

With B3, the data in figures 4,5 and 6 may be directly compared with scattering from a sample with a known, or modeled, cross section, thickness and transmission. For example, a $1 \mathrm{~mm}$ thick sample of $\mathrm{H}_{2} \mathrm{O}$ has a cold neutron cross section of $\approx 1 \mathrm{~cm}^{-1}-\mathrm{sr}^{-1}$ in the SANS regime and a transmission of about 0.5 , for a probability $\mathrm{dS} / \mathrm{d} \Omega$ of roughly $0.05 \mathrm{sr}^{-1}$ and would be difficult to detect under the conditions of the measurements in figure 5 . This comparison serves to emphasize the importance of signal-to-noise for very low $Q$ measurements whether with pinholes or focusing optics.

\section{Figure Captions}

Figure 1. Schematic of a pinhole geometry SANS instrument.

Figure 2. Schematic of a SANS instrument with an ideal focusing optic at the sample position.

Figure 3. Schematic of an ideal ellipsoidal mirror of length $L_{m}$ located to image a source aperture of radius $\mathrm{R}_{1 \mathrm{~m}}$ at one focus onto a detector at the opposite focal point.

Figure 4. Measurement of the main beam profile for a 30-meter pinhole collimation SANS instrument at NIST's Center for Neutron Research. The measurement conditions are described in the text. The data have been normalized by dividing by the beam current at the sample aperture (equation 1 ). Treated in this way, the vertical axis represents the probability of scattering per steradian (see Appendix B for details). Note the more than 5 orders of magnitude signal-to-noise ratio at $Q_{\min }$. The dashed line represents the calculated background expected from Fraunhofer diffraction from the edge of the $1.27 \mathrm{~cm}$ diameter cadmium aperture at the sample position. 
373 Figure 5. Measurements of the circularly averaged beam profile on the detector for a $9 \AA$,

374 pinhole-collimated, beam $\left(2 R_{1 p}=14 \mathrm{~mm}, 2 R_{2 p}=3.0 \mathrm{~mm}, L_{1} \approx L_{2}=15 \mathrm{~m}\right)$ compared with the

375 profiles of beams reflected from fully illuminated, $30 \mathrm{~cm}$ long $\times 5 \mathrm{~cm}$ wide, zerodur cylindrical

376 mirrors as described in the text. The data have been normalized by dividing by the beam

377 current at the sample aperture (equation 1). Treated in this way, the vertical axis represents

378 the probability of scattering per steradian (see Appendix B for details). The non-specular

379 scattering from the better mirror is within a factor of 2 of the parasitic scattering observed for

380 the pinhole collimation. This scattering is, however, considerably greater than the background

381 expected solely from Fraunhofer diffraction from the $3.0 \mathrm{~mm}$ sample aperture, which is shown

382 as the dashed curve.

383 Figure 6. Measurements of the circularly averaged beam profile on the detector for a $9 \AA$, 384 pinhole-collimated, beam ( $\left.2 R_{1 p}=14 \mathrm{~mm}, 2 R_{2 p}=3.0 \mathrm{~mm}, L_{1} \approx L_{2}=15 \mathrm{~m}\right)$ compared with the 385 profile from the focusing lens system used for the lowest-Q measurements on the NG-7 30-m 386 SANS instrument at the NCNR [5]. Also shown are the data from Fig. 5 for the profile from the 387 cylindrical mirror with $3 \AA$ rms surface roughness. The parasitic scattering from the lens system 388 and the $3 \AA$ rms mirror is nearly the same. 


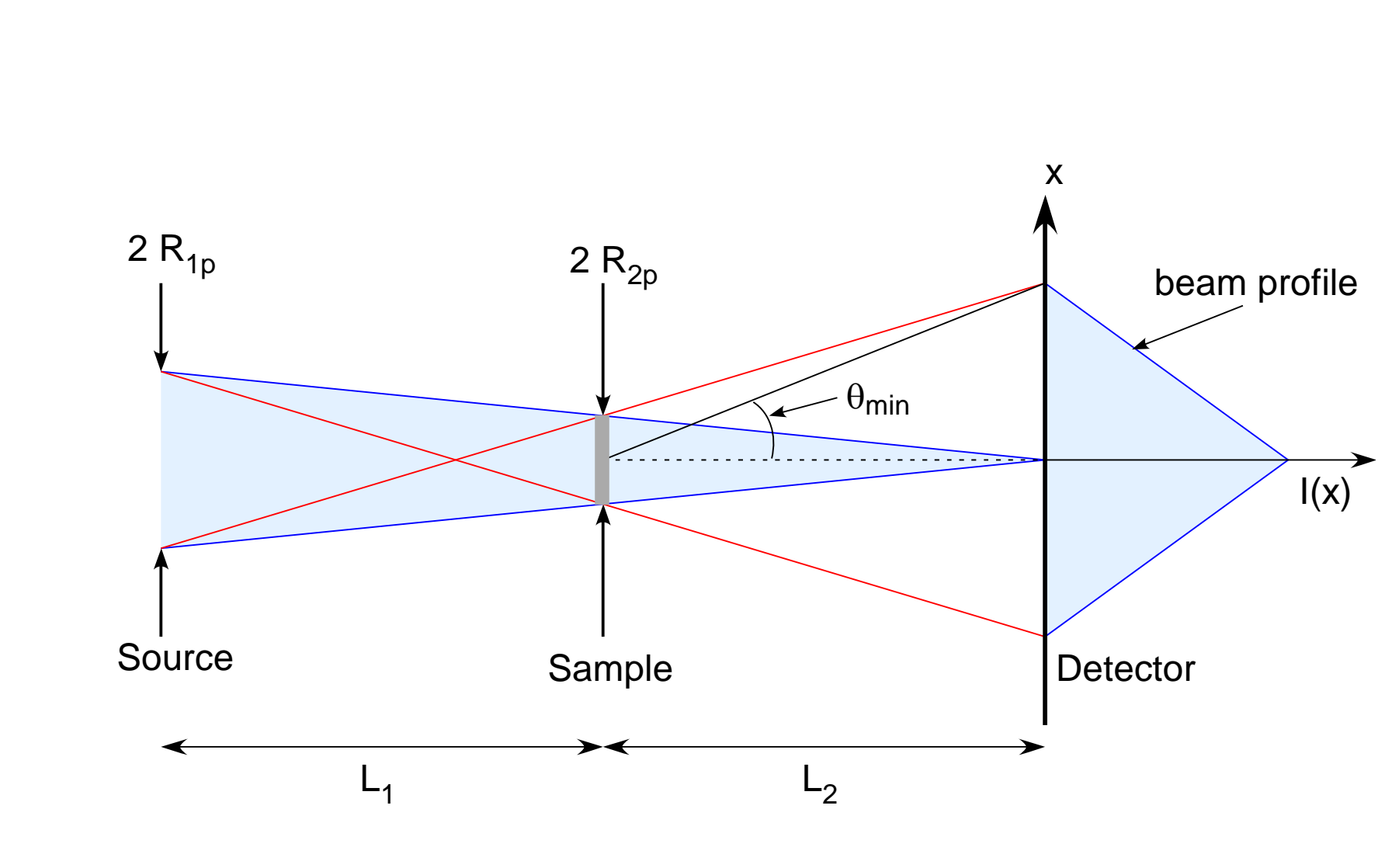

Figure

.

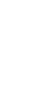

(

.

( 


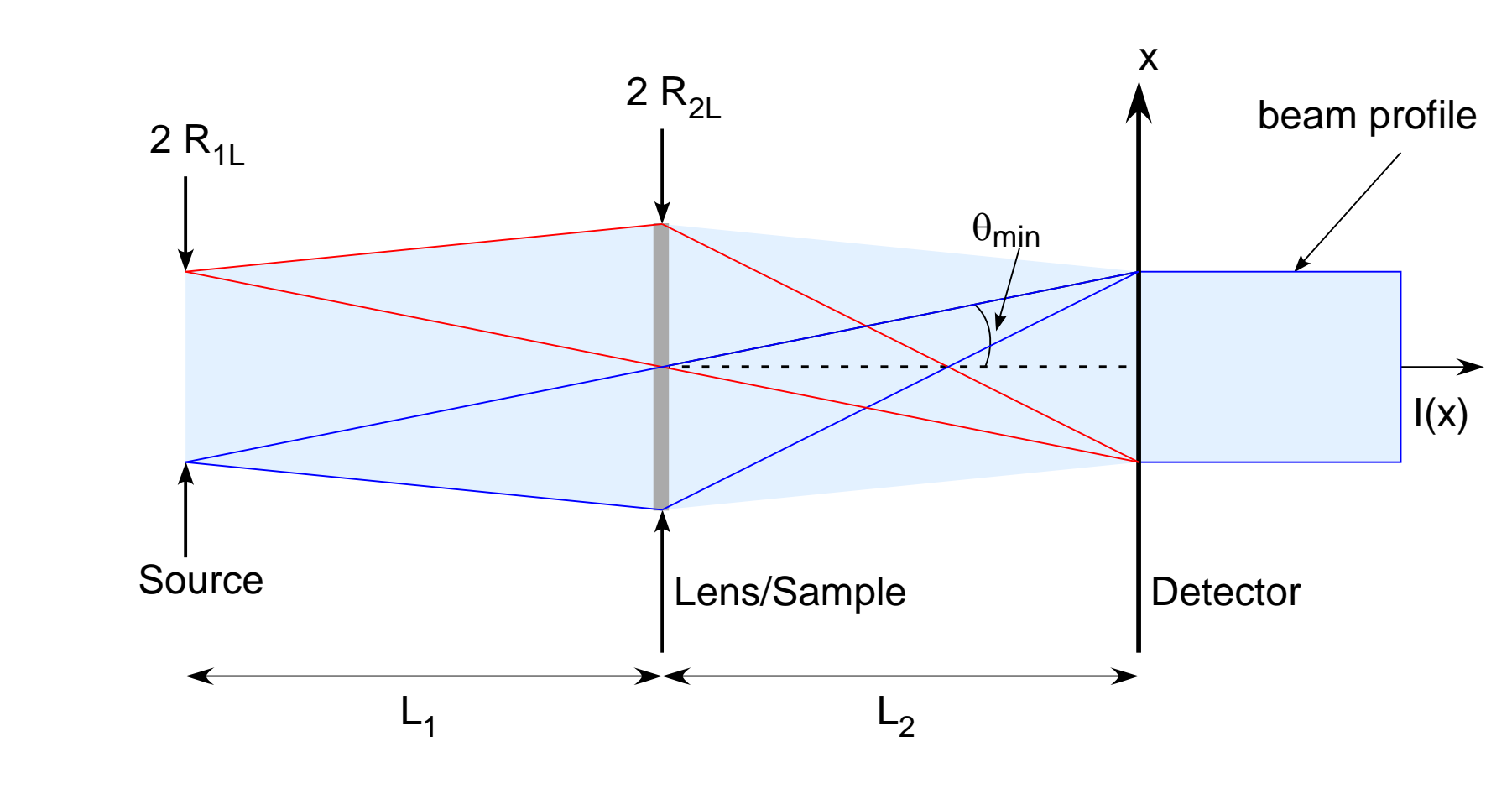

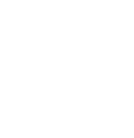

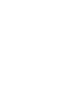
(1)

ra 


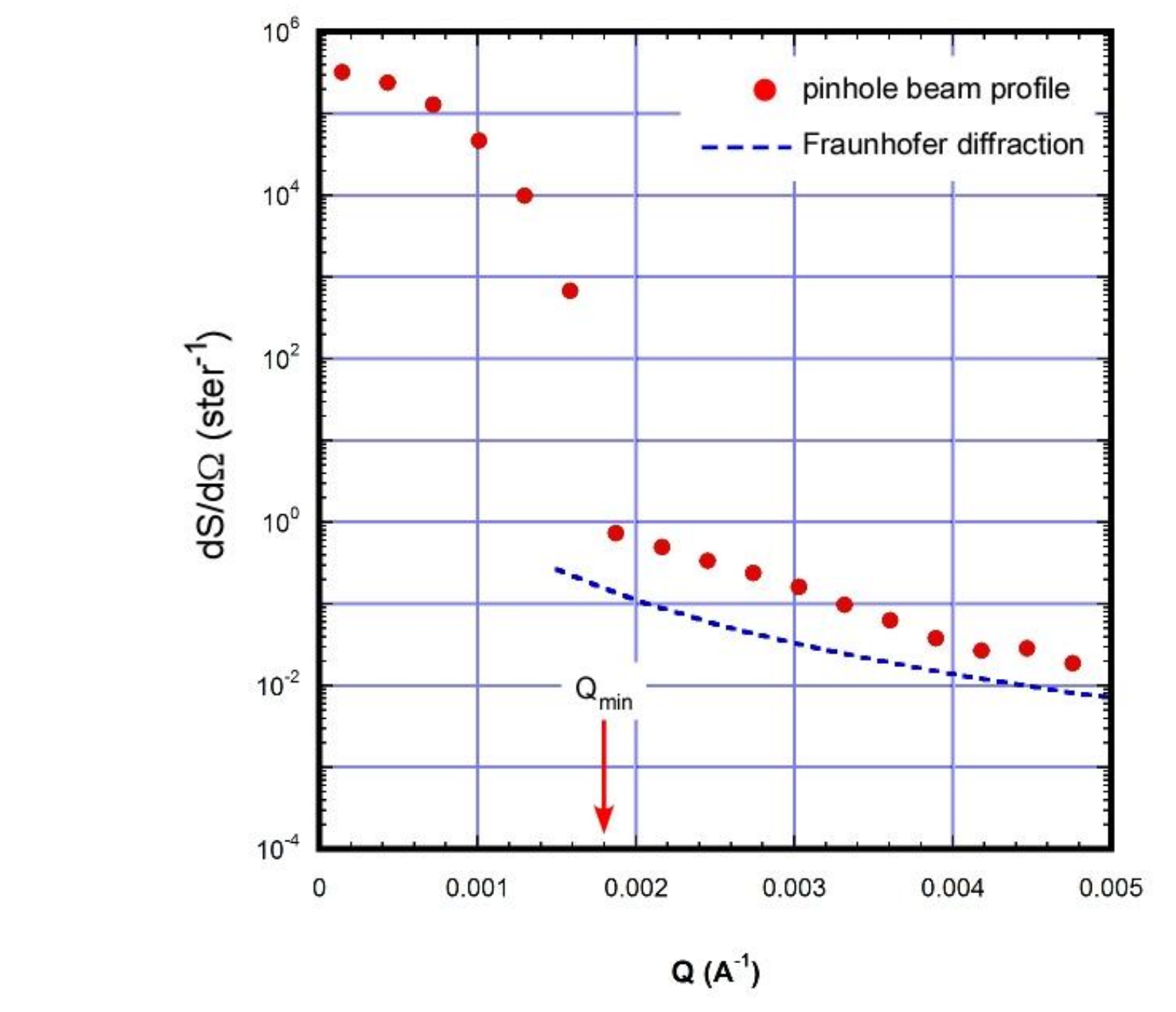

Figure

.

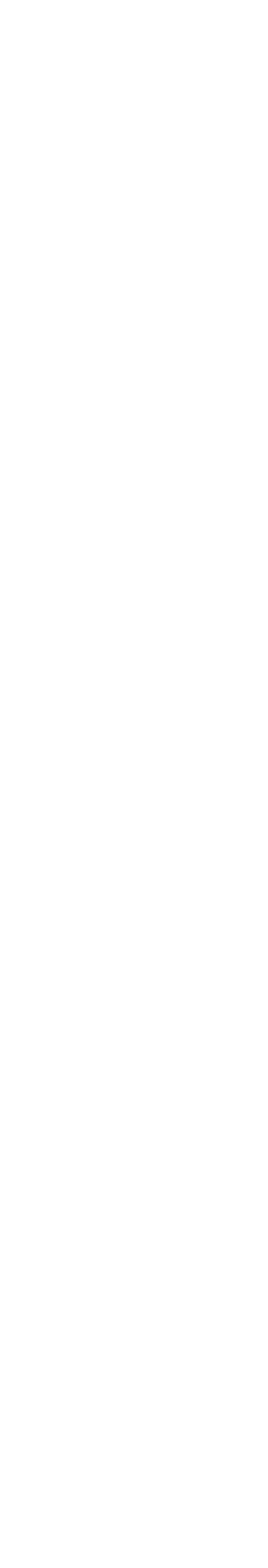




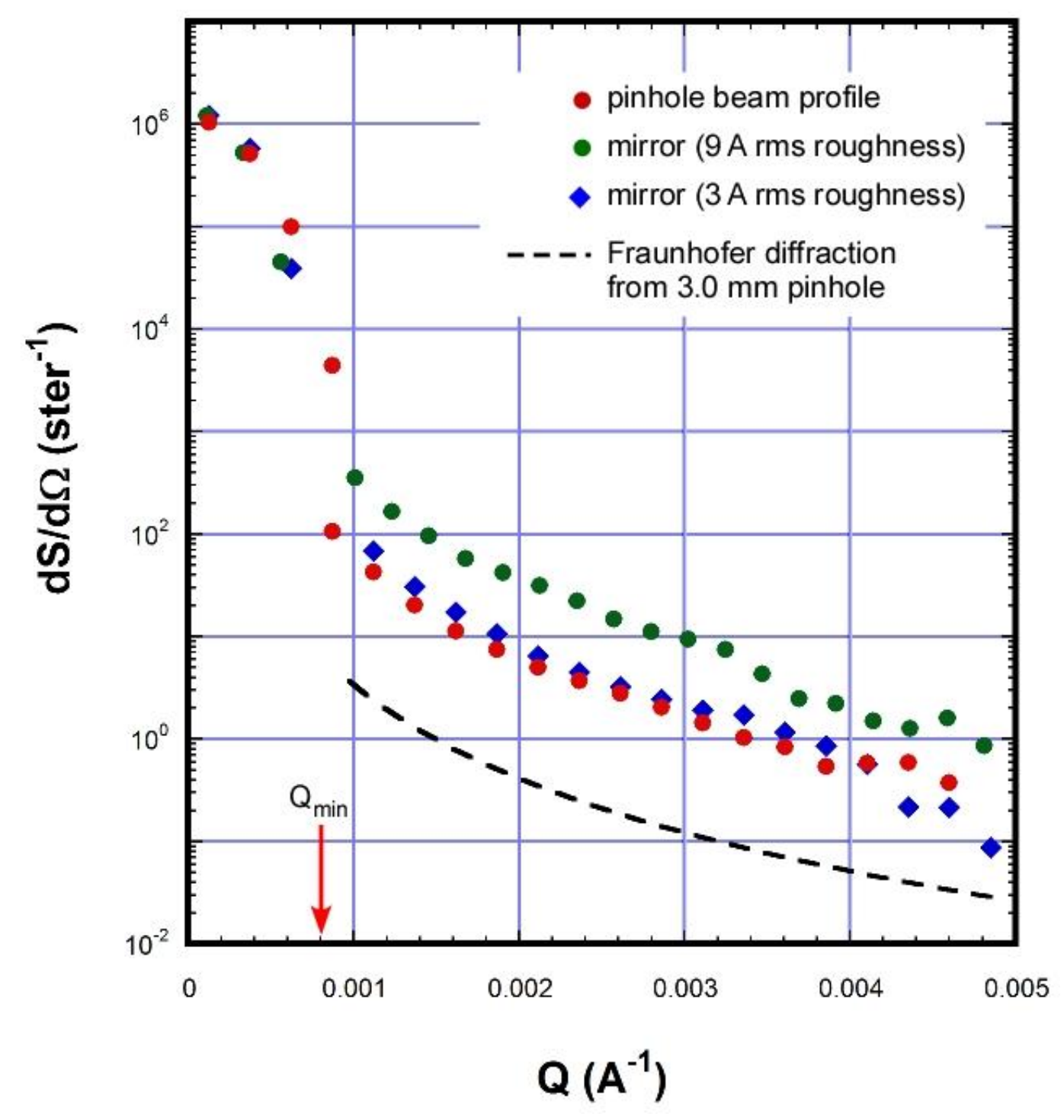

Figure 


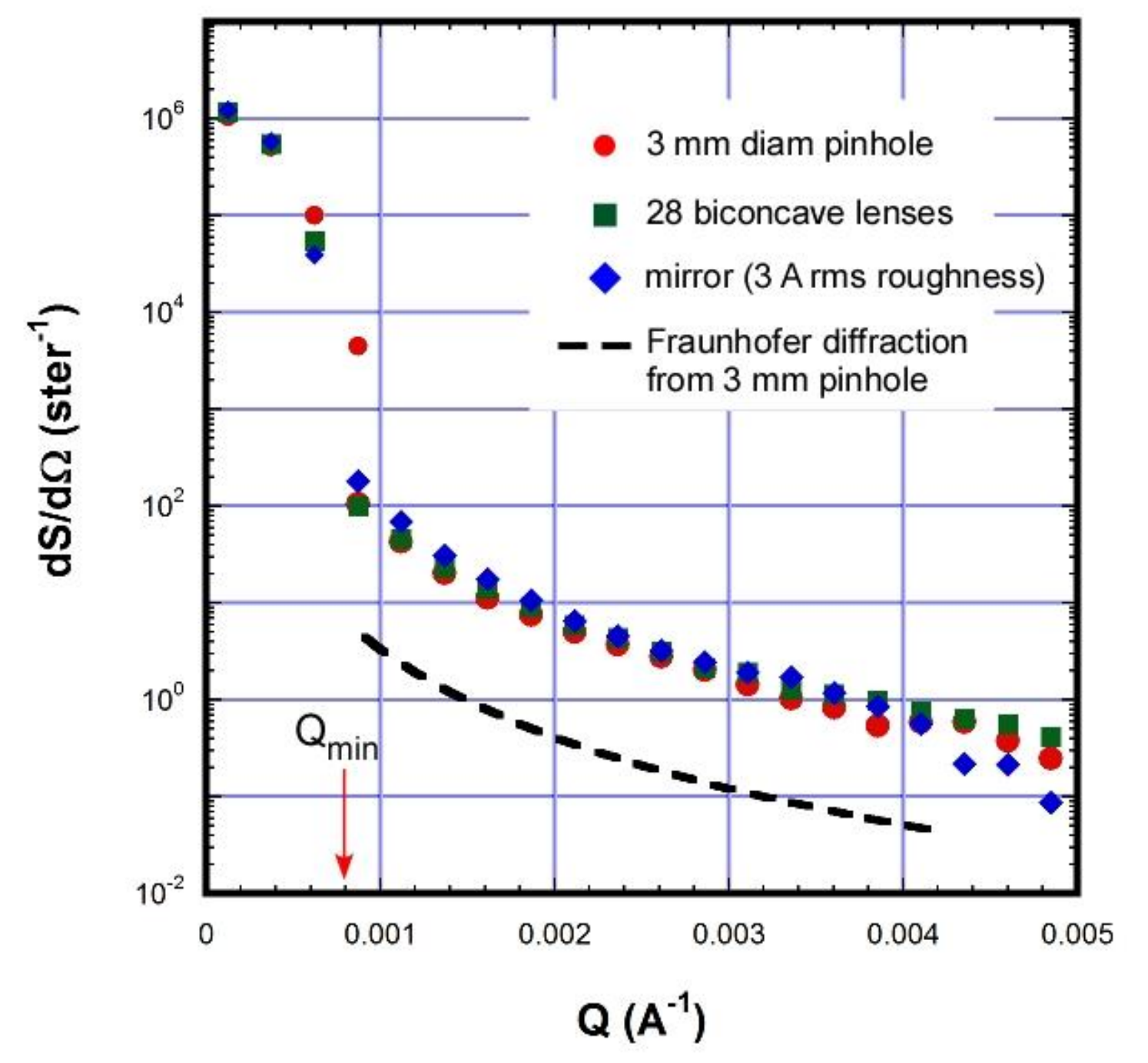

Figure

$Q(A)$ 